\title{
An inexpensive, efficient and safe regimen containing Pasiniazid for MDR-TB in high Tuberculosis epidemic areas - A prospective study in China
}

\section{Wenwen Sun}

Tongji University Affiliated Shanghai Pulmonary Hospital

\section{Qin Tang}

Tongji University Affiliated Shanghai Pulmonary Hospital Jie Wang

Tongji University Affiliated Shanghai Pulmonary Hospital Jinhui Yang

Tongji University Affiliated Shanghai Pulmonary Hospital

Fangyou Yu

Tongji University Affiliated Shanghai Pulmonary Hospital

Hua Yang

Tongji University Affiliated Shanghai Pulmonary Hospital

Lin Fan ( $\nabla$ fanlinsj@163.com )

Tongji University Affiliated Shanghai Pulmonary Hospital https://orcid.org/0000-0002-9411-496X

\section{Research}

Keywords: MDR-TB, Pasiniazid, Treatment success, injectable agents

Posted Date: October 28th, 2020

DOI: https://doi.org/10.21203/rs.3.rs-96887/v1

License: (c) (1) This work is licensed under a Creative Commons Attribution 4.0 International License. Read Full License 


\section{Abstract}

Background: MDR-TB $\triangle$ multidrug-resistant tuberculosis $\triangle$ remains the challenge with low success rate of treatment. Pasiniazid had been applied in China for two decades, but relevant clinical studies are rare. To verify the efficacy and safety of a regimen containing pasiniazid on MDR-TB, a prospective clinical study was conducted in China.

Methods: Patients with MDR-TB satisfied with inclusion criteria were prospectively enrolled into the study from 2017 June to $2018 \mathrm{Dec}$, given the regimen and followed up, observed the treatment outcome and adverse effect of drugs made up the regimen.

Results: A total of 114 patients diagnosed as MDR Pulmonary tuberculosis(MDR-PTB) were enrolled into the study and given the regimen with six months of Capremycin $(\mathrm{Cm})$, Levofloxacin(Lfx), Cycloserine(Cs), Protionamide(Pto), Pyrazinamide(Z) and Pasiniazid $\triangle \mathrm{Pa} \mathbb{Q}$, followed by 12 months of LfxCsPtoZPa. The overall treatment success rate in all enrolled patients was $79.8 \%(91 / 114)$ while it was significantly higher in newly treated MDR-PTB $(91.7 \%, 33 / 36)$ than that in retreated MDR-PTB $74.4 \% \varangle 58 / 78, p$ value was 0.03); Patients infected with strains resistant to fluoroquinolones impacted the treatment outcome $(P<0.05)$ while other drugs did not $(P>0.05)$. Pasiniazid was taken safely without adverse reaction during the course of treatment.

Conclusions: The long term of anti-MDR-PTB regimen containing Pasiniazid was proved to be effective, safe and inexpensive although it contained injectable agents. This regimen is suited to be applied in areas with poor resource and high TB burden.

\section{Introduction}

Multidrug-resistant tuberculosis (MDR-TB) remains great challenge for TB control. According to the update report there were half a million new cases of rifampicin-resistant TB (RR-TB)in 2018 worldwide,of which $78 \%$ had MDR- TB(1). The treatment on MDR-TB had current status with low success rate of treatment, long course, low tolerance of patients and high cost. The global success rate of MDR/RR-TB was only as low as $56 \%$, although success rate was varied in different countries or regions which depends on different factors such as economical support, management, regimens, drug quality, newly drug included, compliance and tolerance of patients. How achieves the optimum outcome of treatment with high safety for MDR-TB became the best important for clinicians.

WHO guidelines on the treatment of MDR/RR-TB in 2019 and 2016 recommended the long term regimens and short term regimens $(2,3)$, the long term regimen for MDR-TB belongs to the traditional treatment strategy while short term regimen has indications such as excluding resistance to fluoroquinolones or second-line injectable agents. A short, inexpensive regimen study was published including gatifloxacin, clofazimine, ethambutol, and pyrazinamide throughout the treatment for 9 months added by an intensive phase of a minimum of 4 months including prothionamide, kanamycin, and high-dose isoniazid, giving a $87.9 \%$ of cure rate(4). Another study was 12 months of short course regimen including gatifloxacin, 
clofazimine, prothionamide, ethambutol and pyrazinamide throughout the course added by kanamycin and isoniazid as an intensive phase of a minimum of 4 months, giving $89 \%$ of success rate(5). Above studies were implemented in the African counties which included clofazimine, whereas clofazimine was hard to be accepted by most Asian patients due to side effect of skin getting dark. Although one study in China verified a regimen with high treatment success rate of $76.3 \%$ based on molecular DST(drug resistance testing) indicating sensitive to pyrazinamide(6), however, the regimen was not enough to be recommended in most regions of China where there had no condition to have PZA DST.

As recommended by updated WHO guidelines on MDR-TB, fluoquinolones, linezolid and bedaquiline were listed as group A by firstly chosen, cycloserine/terizidone and clofazamine as group B by secondly chosen. More and more studies were published recent years basing on the regimens containing bedaquiline and other new drugs. Bedaquiline can improve the treatment efficacy as one of new anti-TB drugs, getting favorable outcome of $73-80 \%$ of patients with $\operatorname{MDR}-\mathrm{TB}(7,8)$. Bedaquiline combined with delamanid in the regimens revealed safety and efficacy with no obvious prolonged QTc interval(9). Clofazamine had excellent efficacy on the treatment of MDR-TB with favorable $65.1 \%$ of success rate( 10 , 11), linezolid listed as one of the first chosen drugs was proved to be favorable outcome of MDR-TB achieving $69.7 \%$ of cure rate (12).

However, all above drugs had imperfections, new drugs such as bedaquiline and delamanid are too expensive to be afforded by most patients in China according to update status, even linezolid had similar issue with high cost, clofazimine had common side effect of darking the skin which was rejected by most Asian patients, above issues restricted their widely applying in many areas. Pasiniazid is the chemical bonding agents in which p-aminosalicylic acid is combined with the acetylation position of isoniazid, it was mainly applied in the market of Asian country, in china it had been used as second-line drugs against TB for two decades. In Chinese guidelines on MDR-TB $(13,14)$,pasiniazid was listed as one of oral agents for MDR-TB. In the present study, we conducted a prospective study on patients with MDR-TB with an inexpensive regimen containing Pasiniazid according to Chines guidelines on chemotherapy of MDR-TB in order to verify its efficacy and safety.

\section{Methods}

\section{Study patients}

From 2017 June to 2018 Dec, we enrolled patients between 18 and 65 years diagnosed as MDR-TB confirmed by MTB MGIT 960 liquid culture and DST. Inclusive criteria were patients satisfied with the following conditions: patients were diagnosed as pulmonary MDR-TB confirmed by culture and DST at least resistant to isoniazid and rifampicin; patients had no previous history of anti-TB treatment or had previously administered only by first-line drugs or administered by second-line anti-TB drugs no more than one month; patients willing to join the study. Excluded criteria were as follows: patients had been administered by second-line anti-TB drugs more than one months; patients had poor compliance to any drugs within the regimens; had severe cardiovascular, liver, kidney, blood system or other serious 
diseases; coexisted with extrapulmonary tuberculosis; co-infected with HIV and other virus, taking immunosuppressive agents; pregnant or less than 18 years or older than 65 years.

\section{Ethic Statement}

The study was approved by The Ethics Committees of Shanghai Pulmonary Hospital, Tongji University School of Medicine, the approval number was k17-138. Individual participants were gave written informed consent before enrollment into the study. Adverse reaction was monitored by quality control team in the hospital in order to guarantee the most possibility of safety during the trial.

\section{Study Design}

Patients satisfied with included criteria were prospectively enrolled into the study, administered by the regimen including Capremycin(Cm), Levofloxacin(Lfx), Cycloserine(Cs), Protionamide(Pto), Pyrazinamide(PZA) and Pasiniazid(Pa)for six months with injectable $\mathrm{Cm}$ followed by 12 months of oral Lfx or Mfx, Cs, Pto, PZA and Pa. Levofloxacin was replaced by Moxifloxacin (Mfx) if patients cannot tolerate. The doses of drugs were administered according to the recommendation of Chinese guideline on treatment of MDR-TB and WHO guideline 2014(15). Cm was injectable administered by $750 \mathrm{mg} / \mathrm{day}$, LfX was orally $600 \mathrm{mg} /$ day, Mfx $400 \mathrm{mg} /$ day, Cs $250 \mathrm{mg}$ every $12 \mathrm{~h}$ per day at initially 2 weeks and then 250 mg every 8 h per day, Pto 1500 mg/day, Z 1500 mg/day, Pa was at 20 mg/kg/day (from 800 mg/day to $1200 \mathrm{mg}$ /day taken orally after three meals). All included patients were followed up at outpatients department concerning treatment efficacy, side effects of drugs and safety monitoring. Sputum culture was tested once a month and Chest CT once three months. The evaluation of treatment efficacy was based on sputum culture conversion, treatment success rate and lesion absorption on Chest CT. Sputum culture negative conversion was considered when patients had negative results of two consecutive sputum cultures at least 30 days apart. Each patient was administered under directly observed therapy (DOT) throughout the treatment course.

Treatment outcomes was classified into as "cured", "Completed treatment", "Treatment failure", "Defaulted" and "died" according to the WHO guideline(2).

\section{BACTEC MGIT 960 Culture and DST}

All enrolled patients was diagnosed and based on the sputum or BALF (Bronchoalveolar lavage fluid) culture positive by BACTEC Mycobacteria Growth Indicator Tube (MGIT) 960 system and DST, the procedure of BACTEC MGIT 960 culture (Difco/Becton Dickson, USA) and DST were followed by protocols of the kits (BD Biosciences, Sparks, Md.) The DST of drugs included Isoniazid, Rifampicin, Streptomycin,Ethambutol, Ofloxacin, Amikacin, Capremycin. Enrolled patients were tested by routine BACTEC 960 culture and DST by clinical laboratory at the beginning of chemotherapy and culture was performed during the course of the treatment.

\section{Minimum Inhibitory Concentration (MIC) of the drugs that make up the regimen}


Strains from patients were stored and available resuscitated were taken out and cultured, tested by MIC DST of drugs in the regimens including $\mathrm{Cm}$, Lfx(Mfx), Cs, Pto, PZA and Pa. M. tuberculosis H37Rv (ATCC 27294) was used as a control strain. Clinical isolates and H37Rv were initially cultured in the BACTEC MGIT 960 system. Subsequently, $100 \mu \mathrm{L}$ of each MGIT culture was inoculated onto Middlebrook $7 \mathrm{H} 10$ agar (Difco) supplemented with 10\% ADC (5\% bovine serum albumin BSA, $2 \%$ dextrose, $5 \%$ catalase) and incubated at $37^{\circ} \mathrm{C}$ for $4-6$ weeks. Colonies were scraped from $7 \mathrm{H} 10$ agar with visible bacterial growth, inoculated in Middlebrook $7 \mathrm{H} 9$ broth supplemented with 10\% ADC and 0.05\% Tween-80 (Sigma) and incubated at $37^{\circ} \mathrm{C}$ for 3-4 weeks. Capreomycin (Cm Sigma), Cycloserine (Cs, Sigma), Protionamide (Pto, Suzhou Kaiyuan Minsheng Technology Co., Ltd., China) and pyrazinamide (PZA, Sigma) were dissolved in deionized water to make $10 \mathrm{mg} / \mathrm{ml}$ drug solutions. Levofloxacin (Lfx, Sigma), Pasiniazid (Pa, Chongqing Winbond Pharmaceutical Company, China) were dissolved in $0.1 \mathrm{~mol} / \mathrm{L} \mathrm{NaOH}$ and formulated into $10 \mathrm{mg} / \mathrm{ml}$ drug solution. All the drug solutions were filtered with a $0.22 \mu \mathrm{m}$ sterile filter (Millpore Company, USA), and stored at $-70^{\circ} \mathrm{C}$ after separate packing. The final concentrations for each drug respectively diluted with Middlebrook $7 \mathrm{H} 9$ broth supplemented with $10 \% \mathrm{ADC}$ and $0.05 \%$ Tween-80 were $\mathrm{Cm} 0.25 \sim 128 \mu \mathrm{g} / \mathrm{ml}$, Cs $0.25 \sim 128 \mu \mathrm{g} / \mathrm{ml}$, Pto $0.125 \sim 256 \mu \mathrm{g} / \mathrm{ml}, \mathrm{Pa} 0.015 \sim 32 \mu \mathrm{g} / \mathrm{ml}, \mathrm{Lfx} 0.03 \sim$ $64 \mu \mathrm{g} / \mathrm{ml}$ and PZA $12.5 \sim 1600 \mu \mathrm{g} / \mathrm{ml}$. An inoculum of M. tuberculosis from the log phase culture was transferred into sterile bottles containing $2.5 \mathrm{~mL}$ Middlebrook $7 \mathrm{H} 9$ broth and glass beads. The suspension was vortexed to break the clumps for 5-10 minutes, then left to sediment for 10-15 minutes. $2 \mathrm{~mL}$ supernatant was then transferred to sterile flat-bottomed glass test tubes, and the turbidity of the suspension was measured and adjusted to McFarland 1.0. The suspension was then diluted 1:10 for PZA MIC assay and 1:100 for MIC determination of other drugs with Middlebrook 7H9 broth, and then inoculated into the drug-containing 96 -well plates. Meanwhile, $100 \%, 10 \%, 1 \%$ bacterial growth control well for each M. tuberculosis were set up. Plates were sealed and incubated at $37^{\circ} \mathrm{C}$ for 14 days. The 96 well U-shaped plates were placed on an inverted magnifying glass to observe the white bacterial pellets in the bottom of each well and record the results that the presence of visible bacterial pellets is positive, but no visible bacterial pellet is negative. The MIC value was defined as the lowest drug concentration that inhibited growth of bacteria compared with the $10 \%$ (PZA) or 1\% (other drugs) positive control. The MIC value of a strain to certain drug higher than $(\geq)$ the cut-off concentration is resistant to this drug. The cut off MIC values for all tested drugs were showed in Table 1. 
Table 1

Drug concentration range $(\mu \mathrm{g} / \mathrm{mL})$ and the cut off concentration of MTB

\begin{tabular}{|lll|}
\hline & Concentration range $(\mu \mathrm{g} / \mathrm{mL})$ & $\begin{array}{l}\text { Cut-off concentration } \\
(\mu \mathrm{g} / \mathrm{mL})\end{array}$ \\
\hline Capremycin & $2 \sim 128$ & 8 \\
\hline Cycloserine & $0.5 \sim 128$ & 32 \\
\hline Isoniazid & $0.5 \sim 16$ & 0.2 \\
\hline p-aminohydroxyacid & $0.5 \sim 128$ & 2 \\
\hline Pasiniazid & $0.015 \sim 32$ & 1 \\
\hline Moxifloxacin & $0.06 \sim 32$ & 0.5 \\
\hline Levofloxacin & $0.03 \sim 64$ & 1 \\
\hline Protionamide & $0.5 \sim 256$ & 8 \\
\hline Pyrazinamide & $12.5 \sim 1600$ & 200 \\
\hline
\end{tabular}

\section{Treatment Efficacy Evaluation}

The patients were examined by Chest CT imaging every three months and sputum culture once a month during the treatment period. The imaging was evaluated by two radiologists and two pulmonary specialists. "cure" was referred as patients completed treatment with consistently at least five negative culture results for the final 12 months of the treatment course and without evidence of treatment failure; "Completed treatment" was referred as patients completed the treatment according to the programme protocol but did not completed the requirement for bacteriological results; "Treatment failure" was referred as patients had sputum culture positive in the final 12 months of the treatment course or if any one of the final three cultures was positive; "died" was patients died from any reason during the course of anti-TB treatment; "Defaulted" was referred as patients whose TB treatment was interrupted for at least two consecutive months for any reason. Treatment success was defined as sum of cured and treatment completed and called as favorable outcome, unfavorable outcomes included "failure", "default" and "death".

\section{Safety Assessment}

Included patients were monitored by liver and lung function, blood routine testing at least once a months, hearing and vision testing once a month, followed at outpatients department of Tuberculosis by physicians once a moths or less if possible. Physicians can do with adverse reactions during follow up if happened.

\section{Statistical analysis}


Statistical analysis was used SPSS 18.0(IBM Corp, Armonk, NY, USA). Categorical variables such as treatment success rate, sputum conversion rate were analysed using $X^{2}$ tests and Fisher's exact tests, and continuous variables were analysed using independent t-tests and Mann-Whitney U-tests. P value $<0.05$ was considered statistically significant. Multivariate analysis to determine the impact factors of treatment outcome was conducted with logistic regression model.

\section{Results}

\section{Study population}

During the study period, a total of 114 MDR-TB patients satisfied with included criteria participated in the study, including 72 males with median age at 35.7 years (range 19-64 years) and 42 females with median age at 32.2 years (range 19-61 years), included patients had 14 cases with XDR-TB, 36 cases with newly treated MDR-TB, 78 cases with retreated MDR-TB. The general characteristics of included patients was shown in Table 2

Table 2

Clinical characteristics of included patients with MDR-TB

\begin{tabular}{|llll|}
\hline characteristic & Newly treated (36) & Retreated (78) & P value \\
\hline median age & $32.1 \pm 12.4$ & $35.5 \pm 11.5$ & 0.13 \\
\hline Male (\%) & $22(61 \%)$ & $50(64.1 \%)$ & 0.34 \\
\hline median BMI & 19.8 & 19.2 & 0.54 \\
\hline cavities present on Chest CT & & \\
\hline no cavity & $18(50 \%)$ & $36(46.2 \%)$ & 0.82 \\
\hline unilateral & $12(33.3 \%)$ & $35(44.9 \%)$ & 0.08 \\
\hline bilateral & $5(13.9 \%)$ & $7(9.0 \%)$ & 0.14 \\
\hline lesion severity & & & \\
\hline$\geq 3$ fields & & $49(62.8 \%)$ & 0.55 \\
\hline$<3$ fields & $22(61.1 \%)$ & 0.76 \\
\hline complications & $14(38.9 \%)$ & & \\
\hline DM & & $10(12.8 \%)$ & 0.35 \\
\hline
\end{tabular}

\section{Treatment Outcome Of Included Patients}

Of all included patients, the overall rate of treatment success was $79.8 \%(91 / 114)$, among 91 cases with success treatment, $86(75.4 \%)$ cured, $5(4.4 \%)$ completed treatment. 23 cases got unfavorable outcome 
including $10(8.8 \%)$ failures, $10(8.8 \%)$ losing follow up and 1 case $(0.9 \%)$ withdrawing treatment due to intolerance to drugs and 2 cases (1.8) died.

The treatment success rate was different between newly treated and retreated MDR-TB patients.

Treatment success was significantly higher in newly treated MDR-TB $(91.7 \%, 33 / 36)$ than that in retreated MDR-TB(74.4\%,58/78) $\square$ p value was 0.03 . Sputum culture negative conversion rates at the end of the third months and the sixth month were $91.7 \%(33 / 36)$ and $94.4 \%(34 / 36)$ in newly treated group which was significantly higher than that of $69.2 \%(54 / 78)$ at the end of third months and that of $70.5 \%(55 / 78)$ at the end of the sixth month in retreated group, $p$ value was 0.009 and 0.004 , respectively. The culture negative conversion rate of newly treated patients was significantly higher than those in retreated patients, the curve was shown in Fig. 1.

\section{MIC value distributions of related drugs and associated factors with success treatment}

To further verify the effectiveness of regimen from the bacteriological point, we tried to resuscitate the strain of included patients and performed the MIC value of at least six drugs made up the regimen, finally we successfully resuscitated 53 strains of 114 patients included. The cut off value of MIC for Pa was $1 \mu \mathrm{g} / \mathrm{ml}$,MIC varied from $<0.015 \mu \mathrm{g} / \mathrm{ml}$ to $\varangle 32 \mu \mathrm{g} / \mathrm{ml}$, 8 strains had MIC of $\geq 32 \mu \mathrm{g} / \mathrm{ml}, 1$ strain had $8 \mu \mathrm{g} / \mathrm{ml}$ of MIC and 1 stain had $16 \mu \mathrm{g} / \mathrm{ml}, 2$ stains had $4 \mu \mathrm{g} / \mathrm{ml}, 6$ stains had $2 \mu \mathrm{g} / \mathrm{ml}, 8$ strains had $1 \mu \mathrm{g} / \mathrm{ml}, 27$ stains had MIC of $<1 \mu \mathrm{g} / \mathrm{ml}$.

Patients infected with MTB strain resistant to Pa did not impact the treatment outcome, $P$ value was 0.95 . Patients infected with strains resistant to PZA, Pto, $\mathrm{Cs}$ and $\mathrm{Cm}$ did not impact the treatment outcome either, $p$ value was $0.99,0.43$ and 0.74 , respectively. However, OR values of resistance to $\mathrm{Cm}$ and PZA were 1.01 and 1.26 which indicted that resistance to $\mathrm{Cm}$ and PZA had slight likelihood to get unfavorable outcome. Patients infected with strains resistant to FQs did impact the treatment outcome, implying patients with FQs resistance had significantly high likelihood to get unfavorable outcomes, $p$ value was 0.001. The detailed data was shown in Table 3 
Table 3

success treatment and unfavorable outcome associated with drug MIC values

\begin{tabular}{|lllll|}
\hline Clinical factors & Treatment success $(\mathbf{n})$ & $\begin{array}{l}\text { Unfavorable } \\
\text { outcome(n) }\end{array}$ & $\mathrm{RR}(95 \% \mathrm{Cl})$ & $\mathrm{P}$ value \\
\hline PZA resistance & 30 & 7 & $1.01(0.22-4.54)$ & 0.99 \\
\hline Pa resistance & 22 & 5 & $0.96(0.24-3.78)$ & 0.95 \\
\hline Cm resistance & 19 & 5 & $1.26(0.32-5.01)$ & 0.74 \\
\hline Pto resistance & 9 & 1 & $0.42(0.05-3.76)$ & 0.43 \\
\hline FQs resistance & 7 & 9 & $20.77(2.38-181.19)$ & $0.001^{*}$ \\
\hline Cs resistance & 25 & 4 & $0.48(0.12-1.95)$ & 0.30 \\
\hline newly treated & 33 & 3 & $3.79(1.05-13.73)$ & $0.03^{*}$ \\
\hline retreated & 58 & 20 & & \\
\hline
\end{tabular}

\section{Safety And Side Effect Monitoring}

Of included 114 patients, $26.3 \%$ of patients (30/114) had adverse reaction, however, only $10.5 \%$ of patients $(12 / 114)$ had to discontinue the suspected drugs. Of 12 cases with stop taking suspected drugs, 10 cases had to stop taking Capremycin because of tingling, hearing loss or renal functional abnormality, 1 case had to stop taking PZA due to occurrence of gout, 1 case had to stop taking Pto because due to stomachache. The rates of adverse drug reactions were as follows: $\mathrm{Cm} 34.7 \%$, Pto $38.8 \%$, PZA 22.2\%, Lfx 2.0\%, Cs 2.0\%, Pa 0.0\%, it was shown in Fig. 2. The rate of adverse reaction was the most highest in Pto, followed by $\mathrm{Cm}, \mathrm{Z}$. Pa had no occurrence of adverse reaction, indicting its highly safety.

\section{Discussion}

In the present study, we made up a regimen containing Cm, FQs, Cs, Pto, PZA and Pasiniazid for the treatment on MDR-TB based on guidelines of WHO 2016 and Chinese guideline $2015(3,13,14)$. As there was included criteria, we included patients without previous medical history of second-line drugs or no more than one months, therefore patients still can be divided into newly treated patients and retreated patients. The results showed that overall success rate was $79.8 \%$ in which success rates reached as high as $91.7 \%$ in newly-treated patients and $74 \%$ in re-treated patients, the results demonstrated the high efficacy of this regimen against MDR-TB.

Apart from included patients limited in having medical history of first line drugs, the high success rate of these patients was mainly due to the high efficacy and safety regimen by present prospective study. 
Pasiniazid(Pa), the chemical binder of isoniazid and para-aminosalicylic, had excellent activity against MTB and plays a role in the treatment of TB. This drug was successfully developed in 1953 in Italy and launched mainly in Chinese medical market with been as cheap as rifampicin. it was listed as one of group 4 and group C of anti-TB drugs according to the Chinese guideline in 2015 and 2019 respectively $(13,14)$. One study from Chinese patients showed that the fractional inhibitory concentration index (FICl) of pasiniazid combined with Moxifloxacin and rifabutin or rifapentine showed better effect against MDR-TB and XDR-TB(16), it was listed as one of three core drugs for the treatment against retreated TB. A prospective, multicenter, randomized, controlled study in China assessed a 5-month regimen containing Pasiniazid on previously treated patients with pulmonary tuberculosis which showed $74.12 \%$ of treatment success rate and significantly higher than $67.7 \%$ of reference group(17). Another Chinese study showed among $109 \mathrm{INH}$-resistant isolates, only $11.9 \%$ and $19.3 \%$ showed resistance to PAS and Pa respectively (18), therefore $\mathrm{Pa}$ had excellent anti-microbial activity and can been used as effective drug against MTB with resistance to isoniazid(INH) but sensitive to Para-aminosalicyclic(PAS). The MIC values of $\mathrm{Pa}$ in the present study showed that nearly $50 \%$ of MDR-TB strain was sensitive to $\mathrm{Pa}$, resistance to $\mathrm{Pa}$ did not impact the treatment outcome, implying the final effective against MDR-TB depend on synergistic effect of regimen.

The other drugs made up the regimen, $\mathrm{Cs}$ had low resistant rate and Cs containing regimen can achieve a highly successful outcome ( $66 \%$ of cure rate) in China reported by one study (19) and $69.4 \%$ of treatment success rate in another study(20). MIC for $90 \%$ of the recruited stains were below $32 \mu \mathrm{g} / \mathrm{ml}(21)$. Above studies demonstrated the slight side effects of $\mathrm{Cs}$ in the treatment of MDR-TB,the present study also verified its safety and efficacy. Prothionamide(Pto) is the one of the most frequently used second-line drugs against TB $\square$ its propyl-analog is ethionamide(Eto), which had enzymatic activation by mycobacterial EthA inhibit InhA and subsequently inhibit mycolic acid synthesis of MTB(22), the present study showed that the sensitive rate according to the MIC value was as high as $81.1 \%(43 / 53)$, implying its contribution in the high efficacy of this regimen although the statistical analysis showed Pto resistance did not impact the treatment outcome.

FQs is the most common and essential drugs in the treatment on MDR-TB, WHO and Chinese guideline put FQs the important position as one of the first chosen-drugs, although the present study showed the resistant to FQs was $30.2 \%(16 / 53)$, the favorable outcome was closely associated with the resistant status of FQs,implying the resistance to FQs might be able to decide the outcome of treatment. As the regimen limited the patients to been not previously treated by second-line drugs including FQs, it guaranteed the sensitive rate of FQs. Therefore for patients has previously treated by FQs or had failed treatment history using FQs containing regimen, it should be cautious to select FQs as one of anti-TB regimens $(23,24)$.

Capremycin (Cm) was kicked out in WHO guideline 2019 and 2020, the main evidence was from the published study (25) which demonstrate that $\mathrm{Cm}$ was not favorable to the treatment outcome and had poor safety. However, the present study beginning from 2017 to 2018 showed that only $8.7 \%(10 / 114)$ of patients stopped using $\mathrm{Cm}$ due to side effect, the highly success rate of regimen containing $\mathrm{Cm}$ implied 
that $\mathrm{Cm}$ might be effect against MDR-TB along with other drugs and had good tolerance although inconvenience because of injectable agents, the results also indicted the adverse reaction rate of $\mathrm{Cm}$ was $34.7 \%$, the secondly rate of drugs made up the regimen.

PZA was always controversial in the treatment of MDR-TB and XDR-TB, some studies showed that PZA resistant or not did not impact its anti-microbial activity $(21,26,27)$, however, the high resistant rate of PZA had been considered its application in the future(28). In the present study, we tested PZA resistance by MIC value, the resistance rate was $50.9 \%$. Relative risk of PZA resistance was 1.01 , indicating that PZA might slightly impact the treatment outcome.

The regimen in the present study had the following characteristics: highly efficacy as $91 \%$ of treatment success rate in newly treated MDR-TB patients; using Pasiniazid which was few applied beyond China but proved to be effective and cheap in the treatment of MDR-TB; having safety although included injectable agents demonstrating good tolerance in Asian people for Capremycin; inexpensive due to do not select new anti-TB drugs such as Bedaquiline and Delamanid. Above characteristics had strong implying that this regimen can be applied widely in poor-source areas.

\section{Conclusions}

We developed a regimen containing Pasiniazid with the characteristics of inexpensive, safe and high efficacy which was demonstrated by present clinical study in China, having value of recommendation for MDR-TB in poor-source areas with high TB burden.

\section{Abbreviations}

MDR-TB囚multidrug-resistant tuberculosis

Cm®Capremycin

Lfx囚Levofloxacin

Cs $₫$ Cycloserine(

Pto®Protionamide

PZA囚Z囚冈Pyrazinamide

Pa『Pasiniazid

RR-TB囚rifampicin-resistant TB

BALF $\triangle$ Bronchoalveolar lavage fluid

MGIT: Mycobacteria Growth Indicator Tube 
DST: drug resistance testing

DOT: directly observed therapy

XDR-TB Extensively drug-resistant tuberculosis

PAS: para-aminosalicyclic

Eto: Ethionamide

FQs: Fluoroquinolones

\section{Declarations}

\section{Competing interests}

The authors declare that they have no competing interests.

\section{Acknowledgements}

We thank all participants for their time and efforts.

\section{Date available statement}

All data regarding the included participants and laboratory DST data during the study are available from the corresponding author by email request.

\section{Author contribution Statement}

WS: included the patients, treated and followed up the patients, wrote the manuscript; QT:included the

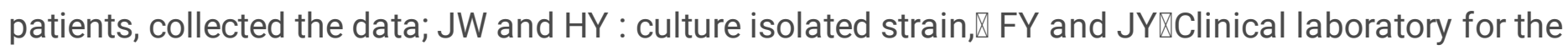
work of MGIT 960 culture; LF: Research design, included the patients, data collection and revised the manuscript. All authors read and approved the final submitted version.

\section{Author information:}

Affiliations

Shanghai Clinical Research Center for Tuberculosis, Shanghai Key Lab of Tuberculosis, Department of Tuberculosis, Shanghai Pulmonary Hospital, Tongji University School of Medicine, Shanghai, China区 Wenwen Sun, Qin Tang and Lin Fan

Shanghai Clinical Research Center for Tuberculosis, Shanghai Key Lab of Tuberculosis, Shanghai Pulmonary Hospital, Tongji University School of Medicine: Jie Wang and Hua Yang 
Shanghai Clinical Research Center for Tuberculosis, Shanghai Key Lab of Tuberculosis, Department of clinical laboratory, Shanghai Pulmonary Hospital, Tongji University School of Medicine: Jinghui Yang, and Fangyou Yu

\section{Corresponding authors}

Correspondence to Lin Fan

\section{Funding acknowledgements:}

This work was supported by the grant from the Shanghai Natural Science Foundation (Grant No.20ZR1446700). The funders had no role in study design, data collection and analysis, decision to publish, or preparation of the manuscript.

\section{Ethics declaration and consent to participate}

This prospective study was approved by The Ethics Committee of the Shanghai Pulmonary Hospital (approval number: K17-138). Each participant or their legal representatives gave written informed consent before enrollment. The name of the ethics committee that approved the study included Lei Zhang, Peng Zhang, Zhongshun Zhang, Haiqing Chu.

\section{Consent for publication section}

Not applicable

\section{References}

1. WHO. Global Tuberculosis Report 2019. Geneva: World health organization: 2019;2019(License):CCBY-NC-SA3.

2. WHO. WHO consolidated guidelines on drug-resistant tuberculosis treatment. Genevia: World Health Organization; 2019.

3. WHO. WHO treatment guidelines for drugresistant tuberculosis 2016 update. Genevia: World Health organization; 2016.

4. A VD, AK M, PK MAS, MR D. S, P D, et al. Short, highly effective, and inexpensive standardized treatment of multidrug-resistant tuberculosis. Am J Respir Crit Care Med. 2010;182(5):684-92.

5. C K, HL JN, N A-K R, JL AF AT. High effectiveness of a 12-month regimen for MDR-TB patients in Cameroon. The international journal of tuberculosis lung disease: the official journal of the International Union against Tuberculosis Lung Disease. 2015;19(5):517-24.

6. Sun F, Li Y, Chen Y, Guan W, Jiang X, Wang X, et al. Introducing molecular testing of pyrazinamide susceptibility improves multidrug-resistant tuberculosis treatment outcomes: a prospective cohort study. The European respiratory journal. 2019;53(3). 
7. Guglielmetti L, Jaspard M, Le Dû D, Lachâtre M, Marigot-Outtandy D, Bernard C, et al. Long-term outcome and safety of prolonged bedaquiline treatment for multidrug-resistant tuberculosis. Eur Respir J. 2017;49(3):1601799.

8. Ndjeka N, Schnippel K, Master I, Meintjes G, Maartens G, Romero R, et al. High treatment success rate for multidrug-resistant and extensively drug-resistant tuberculosis using a bedaquiline-containing treatment regimen. Eur Respir J. 2018;52(6):1801528.

9. Ferlazzo G, Mohr E, Laxmeshwar C, Hewison C, Hughes J, Jonckheere S, et al. Early safety and efficacy of the combination of bedaquiline and delamanid for the treatment of patients with drugresistant tuberculosis in Armenia, India, and South Africa: a retrospective cohort study. Lancet Infect Dis. 2018;18(5):536-44.

10. Duan H, Chen X, Li Z, Pang Y, Jing W, Liu P, et al. Clofazimine improves clinical outcomes in multidrug-resistant tuberculosis: a randomized controlled trial. Clin Microbiol Infect. 2019;25(2):1905.

11. Y D, C Q, X C, J W, W J, H P, et al. Treatment outcome of a shorter regimen containing clofazimine for multidrug-resistant tuberculosis: a randomized control trial in China. Clin Infect Dis, 2020,71(4):1047-1054.

12. Tang S, Yao L, Hao X, Zhang X, Liu G, Liu X, et al. Efficacy, safety and tolerability of linezolid for the treatment of XDR-TB: a study in China. Eur Respir J. 2015;45(1):161-70.

13. Chinese Anti-tuberculosis Association. the guideline of chemotherapy on MDR-TB in China. 2015;2015.

14. Chinese Anti-tuberculosis Association. the guideline of chemotherapy on MDR-TB. 2019;2019.

15. WHO. companion handbook to the WHO guidelines for the programmatic management of drugresistant tuberculosis. Geneva, World health organization; 2014.

16. Zhang L, Yang H, Xiao H, Lu J, Sha W, Zhang Q. Determination of in vitro synergy by a checkerboard method when 3 core antimicrobial agents of the retreatment new scheme combined against MDRMTB and XDR-MTB. Zhonghua jie he he hu xi za zhi. 2016;39(6):464-8.

17. Yan L, Kan X, Zhu L, Xu K, Yin J, Jie L, et al. Short-course Regimen for Subsequent Treatment of Pulmonary Tuberculosis: A Prospective, Randomized, Controlled Multicenter Clinical Trial in China. Clinical therapeutics. 2018;40(3):440-9.

18. Li G, Zhang J, Jiang Y, Zhao L, Liu H, Li M, et al. Cross-resistance of isoniazid, para-aminosalicylic acid and pasiniazid against isoniazid-resistant Mycobacterium tuberculosis isolates in China. Journal of global antimicrobial resistance. 2020;20:275-81.

19. Wang J, Pang Y, Jing W, Chen W, Guo R, Han X, et al. Efficacy and safety of cycloserine-containing regimens in the treatment of multidrug-resistant tuberculosis: a nationwide retrospective cohort study in China. Infection drug resistance. 2019;12:763-70.

20. Li Y, Wang F, Wu L, Zhu M, He G, Chen X, et al. Cycloserine for treatment of multidrug-resistant tuberculosis: a retrospective cohort study in China. Infection Drug Resistance. 2019;12:721-31. 
21. Yu X, Zeng X, Shi W, Hu Y, Nie W, Chu N, et al. Validation of Cycloserine Efficacy in Treatment of Multidrug-Resistant and Extensively Drug-Resistant Tuberculosis in Beijing, China. Antimicrobial agents and chemotherapy. 2018;62(3).

22. Thee S, Garcia-Prats A, Donald P, Hesseling A, Schaaf H. A review of the use of ethionamide and prothionamide in childhood tuberculosis. Tuberc (Edinb Scotl). 2016;97:126-36.

23. Desikan P, Panwalkar N, Chaudhuri S, Khan Z, Punde R, Pauranik A, et al. Burden of baseline resistance of Mycobacterium tuberculosis to fluoroquinolones and second-line injectables in central India. Trans R Soc Trop Med Hyg. 2020;114(4):249-54.

24. Kabir S, Tahir Z, Mukhtar N, Sohail M, Saqalein M, Rehman A. Fluoroquinolone resistance and mutational profile of gyrA in pulmonary MDR tuberculosis patients. BMC pulmonary medicine. 2020;20(1):138.

25. Ahmad N, Ahuja S, Akkerman O, Alffenaar J, Anderson L, Baghaei P, et al. Treatment correlates of successful outcomes in pulmonary multidrug-resistant tuberculosis: an individual patient data metaanalysis. Lancet. 2018;392(10150):821-34.

26. Park S, Jo K, Shim T. Treatment outcomes in multidrug-resistant tuberculosis according to pyrazinamide susceptibility. Int J Tuberc Lung Dis. 2020;24(2):233-39.

27. Kuhlin J, Smith C, Khaemraev A, Tigay Z, Parpieva N, Tillyashaykhov M, et al. Impact of pyrazinamide resistance on multidrug-resistant tuberculosis in Karakalpakstan, Uzbekistan. Int $\mathrm{J}$ Tuberc Lung Dis. 2018;22(5):544-50.

28. Naluyange R, Mboowa G, Komakech K, Semugenze D, Kateete D, Ssengooba W. High prevalence of phenotypic pyrazinamide resistance and its association with pncA gene mutations in Mycobacterium tuberculosis isolates from Uganda. PloS one. 2020;15(5):e0232543.

\section{Figures}




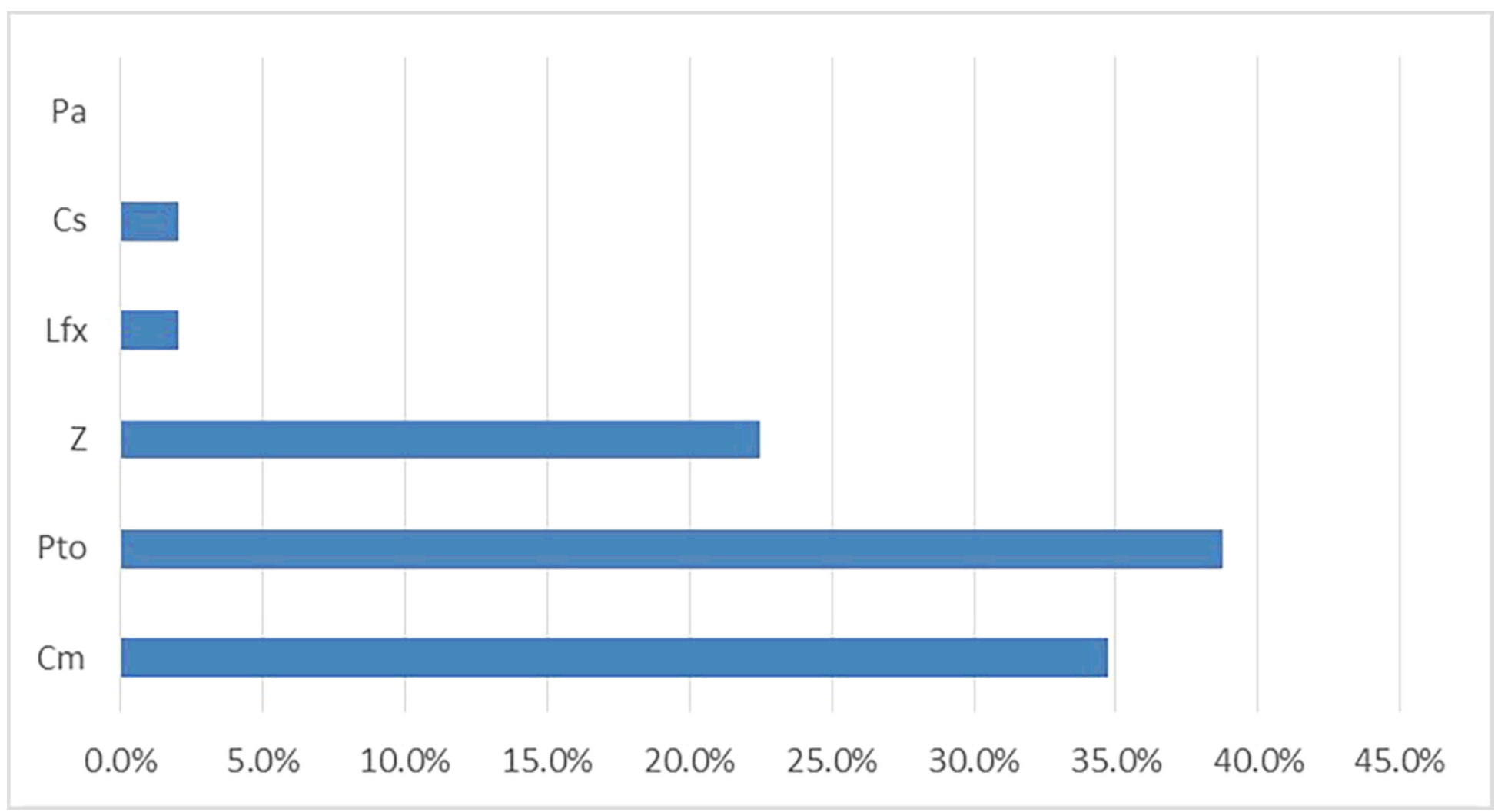

Figure 1

The culture negative conversion rate of newly treated patients was significantly higher than those in retreated patients, the curve was shown in figure 1.

Time to culture conversion months between two groups

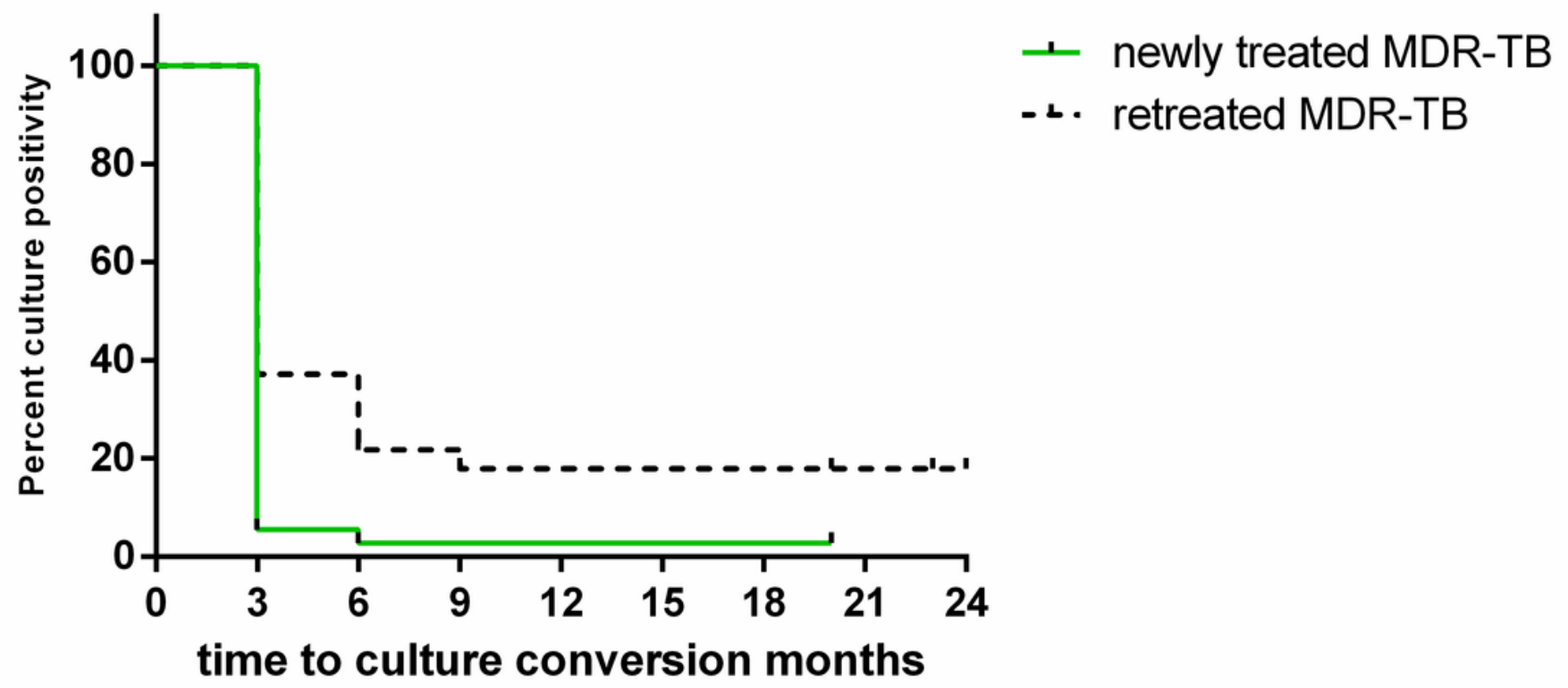

Figure 2 
Rate of adverse drugs reactions

Page 17/17 